\title{
UNIVERSITYOF
}

FORWARD

THINKING

WESTMINSTER用

WestminsterResearch

http://www.westminster.ac.uk/westminsterresearch

Proactive Energy-Efficiency: Evaluation of Duty-Cycled MAC

Protocols in Wireless Sensor Networks

Udoh, E. and Getov, Vladimir

This is a copy of the author's accepted version of a paper subsequently to be published in the proceedings of CITS 2018: The 2018 International Conference on Computer, Information and Telecommunication Systems, Colmar, France, 11 to 13 July 2018, IEEE.

The final published version will be available online at:

https://doi.org/10.1109/CITS.2018.8440194

(C) 2018 IEEE . Personal use of this material is permitted. Permission from IEEE must be obtained for all other uses, in any current or future media, including reprinting/republishing this material for advertising or promotional purposes, creating new collective works, for resale or redistribution to servers or lists, or reuse of any copyrighted component of this work in other works.

The WestminsterResearch online digital archive at the University of Westminster aims to make the research output of the University available to a wider audience. Copyright and Moral Rights remain with the authors and/or copyright owners.

Whilst further distribution of specific materials from within this archive is forbidden, you may freely distribute the URL of WestminsterResearch: ((http://westminsterresearch.wmin.ac.uk/)).

In case of abuse or copyright appearing without permission e-mail repository@westminster.ac.uk 


\section{Proactive Energy-Efficiency: Evaluation of Duty-Cycled MAC Protocols in Wireless Sensor Networks}

\author{
Ekereuke Udoh \\ Distributed and Intelligent Systems Res. Group \\ University of Westminster \\ London, UK \\ w1562173@my.westminster.ac.uk
}

\author{
Vladimir Getov \\ Distributed and Intelligent Systems Res. Group \\ University of Westminster \\ London, UK \\ v.s.getov@westminster.ac.uk
}

\begin{abstract}
Duty cycling happens to be one of the major techniques for conserving energy in wireless sensor networks and this research aims to answer questions with regards to the effect of duty cycles on the energy efficiency as well as the throughput of three duty-cycle protocols - Sensor-MAC (SMAC), Timeout-MAC (TMAC) and TunableMAC. Although other dutycycle protocols are reviewed, the aforementioned three protocols are observed in OMNET++ simulator via the Castalia framework. Graphical results are produced which show the energy consumption and throughput as the duty cycle is varied and the variations in results for each of the three protocols are analyzed. The results provide insight into how to ensure 'proactive energy-efficiency' whereby the impact of denial-ofsleep attacks can be minimized while throughput is maximized.
\end{abstract}

Keywords - duty cycle, MAC protocols, wireless sensor networks, proactive energy-efficiency

\section{INTRODUCTION}

Media access control (MAC) protocols play a huge role in the energy-efficiency of wireless sensor networks (WSN) especially as these networks have resource-constrained devices which are mostly battery powered. The radio is the major source of energy consumption in these devices and access to radio is controlled by the MAC layer. Hence, the MAC protocols use duty cycling as one of the ways for saving energy by making nodes go to sleep when they are idle and having them only wake up when they need to transmit or receive data.

While duty cycling can save energy, it can also negatively affect throughput. This gives rise to the need to experimentally observe the effects of duty cycling on energy consumption and throughput in different MAC protocols to understand which other variables, beyond the duty cycles, could help to improve the energy-efficiency balance.

Understanding the various sources of energy loss such as collision, overhearing, idle listening, and control overhead is important as this would help give insight into what techniques other than duty cycling can help save energy.

Energy loss can be random or can be caused by an intentional attack, one of which is called a denial-ofsleep (DoS) attack [1], [2], [3], [19]. Various methods are used to carry out a DoS attack. These are commonly classified as sleep deprivation, barrage, synchronization, replay, collision and broadcast attacks [4]. These attacks take advantage of vulnerabilities such as frame collisions, message overhearing and idle listening [5]. On the other hand, various approaches have been proposed to detect and prevent DoS attacks. Existing comparisons of these approaches are qualitative in nature with a focus on their strengths and weaknesses [4].

It is pertinent to note that in the context of DoS, a number of approaches exist to curb these attacks, however the majority of them are techniques that do not take energy-efficiency into consideration and even when they do, throughput becomes a trade-off which could become counter-productive in the long run. The most notable existing approaches include Gateway-MAC (GMAC) [9], Hash-based scheme [10], Clustered adaptive rate limiting [3], Fake schedule switch scheme [11], Absorbing Markov chain (AMC) model [12], Secure wakeup scheme [13], Zero knowledge protocol [14] and Cross layer mechanism [15].

The aim of this research therefore is to analyse the effects of duty cycling on energy efficiency and throughput in three MAC protocols and analyse the results to find clues as to how to create "proactive energy efficiency" - energy conservation that still supports throughput while minimising the impact of DoS attacks. Thus, a simulation experiment is carried out based on three duty-cycled protocols to monitor their effect on energy consumption as well as their effect on throughput. The analysis of the 
experimental results is then followed by a recommendation of a model for building an improved proactive energy-efficient protocol. The paper concludes with plans for future work.

\section{Methodology}

\section{A. Existing duty-cycle protocols}

Duty cycling involves a cycle of an active period and a sleep period. However, these schedules need to be synchronised in some way to allow for harmony rather than counter-productivity. Hence, the need to consider several categories of MAC layer protocols [6] as listed below:

- Static scheduled protocols (SMAC);

- Adaptive group schedule (TMAC);

- Adaptive repeated schedule (SCP-MAC);

- Adaptive staggered schedule (DMAC);

- Adaptive reservation schedule (RMAC).

\section{B. Simulation}

The simulation was carried out using OMNET++ and Castalia framework [17]. It involves experiments with SMAC and TMAC which are the most popular among the duty-cycled protocols as well as the TunableMAC protocol, discussed later in Section III. Measurements were performed under different network sizes while observing the energy consumption and reception.

Our simulation experiments are based on a bridge test application whereby the structural health of a bridge is monitored, a 40-metre bridge with 7 nodes and a 200-metre bridge with 34 nodes as well as 1000-metre bridge with 154 nodes. Each node is arranged in the form of a grid is 20 metres apart from the next node, hence this explains the number of nodes which correspond to the length of the bridge. A sample interval of $1000 \mathrm{~ms}$ is used, while the consumed energy is measured in megawatts. To reduce randomness in the results, a random seed value has been used and set to 10 which indicates the number of repetitions of the simulation. The value 10 was determined by using a $95 \%$ confidence interval along with manually checking the smoothness and precision of the results by trying out different seed values and observing the results.

\section{REVIEW OF EXISTING APPROACHES}

\section{A. SMAC (Static-Scheduled)}

The SMAC protocol [16] has a static schedule which is fixed during network setup. This means that nodes have a fixed duty cycle (durations for sleep and listen are fixed). The implications of this is that in low traffic, energy may be wasted during the fixed listen durations whereby nodes will stay awake unnecessarily for the sake of completing the schedule, instead of sleeping.

\section{Algorithm for SMAC}

- Node listens to medium for a certain period by performing Carrier sense (CS).

- If node receives schedule from neighbour, it chooses it and becomes a follower.

- The node broadcasts its new schedule after a random delay.

- Else, the node determines its own schedule and broadcasts it to neighbours.

- $\quad$ Node sends message using Request-to-send (RTS) by randomly selecting a time slot.

- If a node hears an RTS or Clear-to-send (CTS) message, it goes to sleep.

\section{B. TMAC (Adaptive Grouped Schedule)}

Unlike SMAC where the duty cycle is fixed, the TMAC allows for flexibility during a node's listen time according to the traffic density. The minimum time for which a node stays awake before going to sleep is the adaptive timeout (TA). Other packets used by TMAC include Data-send (DS) which is a dummy message sent when a node wants to transmit at the same time it hears a future RTS packet. This helps prevent collision by delaying transmission.

\section{Algorithm for TMAC}

- Nodes wake up at the beginning of the slot.

- Node sleeps if no activity is observed.

- If a node overhears a CTS, it stays awake till the end of the transmission.

- At the end of the transmission, the node contends for the medium again and begins transmission if it wins the medium.

- If a node has pending data, it informs its intended receiver using a future RTS technique to avoid the early-sleeping problem.

- The receiver remains active until the message is received.

- If another node is about to transmit and overhears a future RTS packet, it sends DS to its receiver to delay transmission.

\section{SCP-MAC (Adaptive Repeated Schedule)}

This protocol improves the grouped schedule protocols by eliminating the early sleep problem by creating repeated small active periods in one slot.

\section{Algorithm for SCP-MAC}

- $\quad$ Nodes perform CS by randomly selecting a slot within the first contention window. 
- If channel is idle sender transmits a short wake-up tone timed to intersect with the receiver's channel polling.

- After waking up the receiver, the sender transmits the data packet.

- Else node aborts transmission until next frame.

\section{DMAC (Adaptive Staggered Schedule)}

This works very well with the tree-based topology in such a way that the schedule of one node is synchronised with the schedule of the next hop node.

\section{Algorithm for DMAC}

- Node sends a packet to the next hop node on the route.

- The node awaits acknowledgment and the next hop node enters receiving state at the same time.

- If this next hop node is not the destination node, the node enters sending state to forward the packet.

- Else the final destination has been reached.

- If sender does not receive ACK, it queues the message until the next sending.

- If node has multiple packets to send, it increases its duty cycle and requests other nodes along the sink route to increase their duty cycles too.

\section{E. RMAC (Adaptive Reservation)}

This protocol allows nodes to negotiate their schedules.

\section{Algorithm for RMAC}

- During the Synchronisation (SYNC) phase, nodes synchronise their clocks.

- During the data phase, the sender waits for a randomly chosen time plus an additional DCF Inter-frame Space (DIFS) period.

- If no activity is detected, the sender transmits a Pioneer Control Frame (PION).

- The next hop node along the route looks up the next hop and forwards the PION to it after waiting for a Shortest Inter-frame space (SIFS) period.

- The process continues until destination is reached.

- Data transmission begins during sleep period.

- Each node returns acknowledgement (ACK) after receiving packet and returns to sleep mode.

- Process continues until data is received at destination.

\section{F. TunableMAC}

TunableMAC is a protocol that was provided along with the WSN Framework, Castalia [17].

\section{Algorithm for TunableMAC}

As the name implies, this algorithm is tuneable and allows 12 of its parameters to be tuned. This protocol can simulate many duty-cycling protocols, but it does not support unicast. It uses Carrier-sense multiple access (CSMA) for its transmission, therefore its persistence and backing off policies can be tuned. Its duty cycle can also be tuned as well as the train of beacons that can be used to wake up potential receivers.

\section{SIMULATION RESULTS}

\section{A. Energy Simulation}

This section presents experimental results for energy consumption. This is done first for the TunableMAC and secondly, the three protocols (SMAC, TMAC and TunableMAC) are compared in terms of their energy consumption.

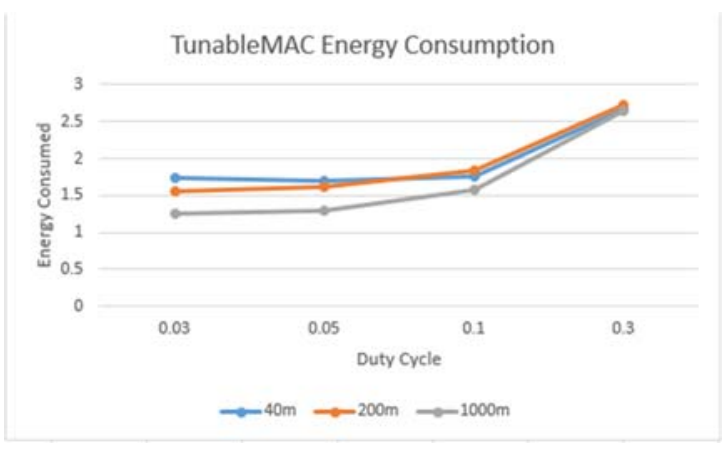

Fig. 1. Energy Consumption for TunableMAC at varied duty cycles and bridge sizes

Figure 1 shows that the energy consumption increases in direct proportion to the duty cycle. The energy consumption is highest in the 200-metre bridge when the duty cycle is 0.3 .

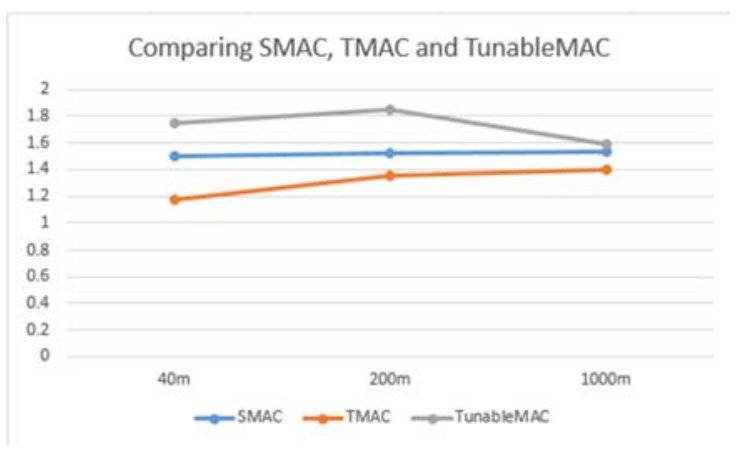

Fig. 2. Energy Consumption for TunableMAC, SMAC and TMAC at varied bridge sizes 
In Figure 2, SMAC appears more stable than TMAC and TunableMAC in terms of energy consumption. TunableMAC consumes the highest energy, followed by SMAC and then TMAC which consumes the least energy.

\section{B. Reception Simulation}

The reception simulation shows the ratio of the number of packets received at the sink to the total number of packets transmitted.

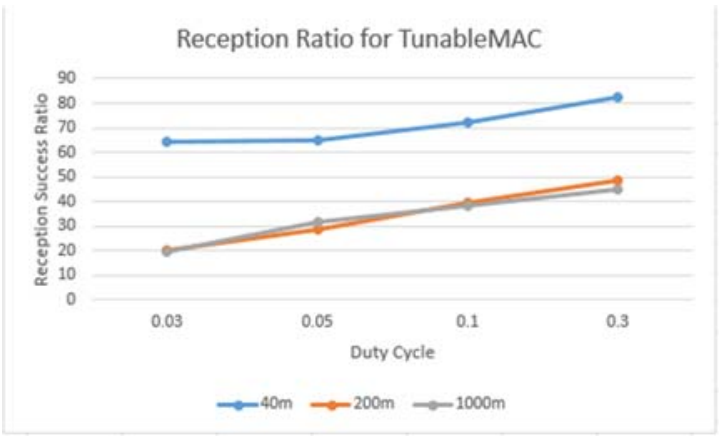

Fig. 3 Reception ratio for TunableMAC at varied duty cycles and bridge sizes

Figure 3 shows that there is a higher reception in the 40 -metre bridge with the $200 \mathrm{~m}$ and $1000 \mathrm{~m}$ competing for a lower reception.

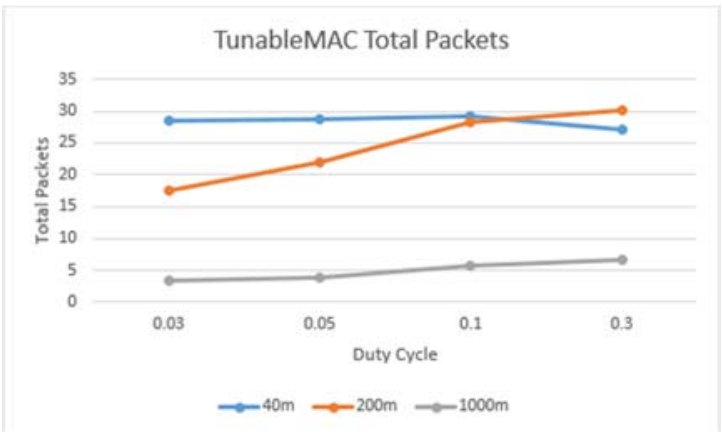

Fig. 4. Total packet for TunableMAC at varied bridge sizes

In Figure 4, more packets are sent in the $40 \mathrm{~m}$ bridge and this is followed closely by the $200 \mathrm{~m}$ bridge and then the $1000 \mathrm{~m}$ bridge takes the last position with relatively low number of packets transmitted.

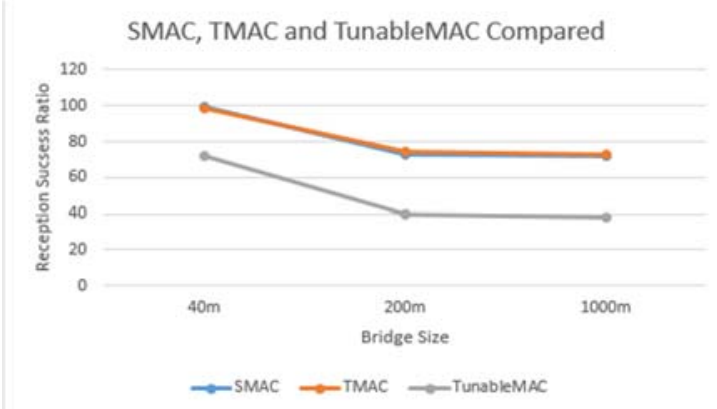

Fig. 5. Reception ratio for TunableMAC, SMAC and TMAC at varied bridge sizes
In Figure 5, TMAC and SMAC have similar reception ratios while TunableMAC has a lower reception ratio.

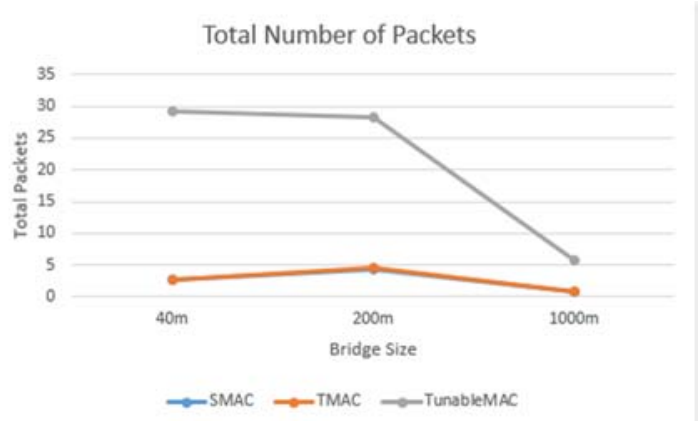

Fig. 6. Total packets for TunableMAC, SMAC and TMAC at varied bridge sizes

In Figure 6, TunableMAC has the highest number of packets followed by SMAC and TMAC.

\section{ANALYSIS}

Figure 1 shows the changes in TunableMAC energy consumption as the duty cycles change. Energy is most consumed in the $40 \mathrm{~m}$ bridge in total but at duty cycle 0.3 , the $200 \mathrm{~m}$ bridge consumes the highest energy. Energy consumption increases proportionally to the duty cycle. Figure 2 compares SMAC, TMAC and TunableMAC and of all the three TunableMAC consumes the most energy. Figure 3 shows that reception is highest in a $40 \mathrm{~m}$ bridge because of the distance. In Figure 4, throughput is highest in the $40 \mathrm{~m}$ bridge although the $200 \mathrm{~m}$ bridge seems to take over as duty cycle approaches 0.3. In Figure 5, SMAC and TMAC have a better reception ratio than TunableMAC, however in Figure 6, throughput is highest in TunableMAC. TunableMAC appears to spend a lot of energy without much productivity. On the other hand, TMAC and SMAC save more energy but transmit a very small number of packets.

\section{PROPOSED APPROACH}

Based on the above results and analysis, the following issues were identified:

- Too much energy consumption with less productivity. This is evident in the TunableMAC protocol which relatively consumes a lot of energy as shown in Figure 2 but has a relatively low reception ratio.

- Low energy consumption with little or no adaptability to a topological change.

In response to these issues, we propose a novel model based on the virtual clusters approach that is secure and proactively energy-efficient. 


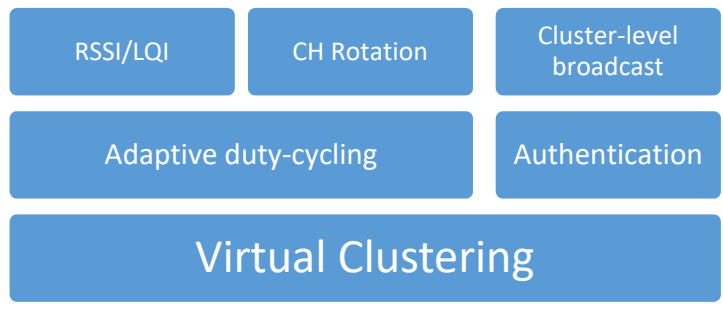

Fig. 7. Conceptual model of components of proposed proactive energy-efficient MAC protocol

The use of virtual rather than real clusters [18] is better in the sense that it is adaptable to any change in topology. Also, there'll also be rotation of cluster heads depending on their availability and resource consumption. Secondly, an adaptive duty cycling would then be incorporated into the virtual clusters. Thirdly, a measurement-based security technique such as RSSI can then be introduced to protect against any form of jamming. The presence of virtual clusters also be utilised to only allow clusterlevel broadcasts rather than network-wide broadcasts.

\section{CONCLUSION AND FUTURE WORK}

One of the benefits of this research is that it shows the effect of duty cycling in MAC protocols and their effect on energy consumption. The interesting part is in the TunableMAC protocol which allows tuning of the duty cycle to see the energy levels.

While it is obvious that energy consumption is reduced with lower duty cycles, it is also important to know the cost involved in saving energy with regards to throughput and latency. This raised the question as to what degree of throughput is being traded to save energy. One way to answer this question was to measure the report reception (throughput) in the simulator. The results reveal that although SMAC and TMAC perform better than TunableMAC in terms of energy consumption, TunableMAC outshines them in terms of packet reception (throughput).

In our future work, we plan to on how to adapt TunableMAC by extending the concepts presented in Section VI and Figure 7. An impact analysis will be done on the other higher layers to analyse the overall energy-efficiency and throughput caused by changes to the MAC layer.

\section{ACKNOWLEDGEMENT}

This work was partially supported via a doctoral research scholarship grant by the University of Westminster.

\section{REFERENCES}

[1] T. Bhattasali, "Sleep Deprivation Attack Detection in Wireless Sensor Network," Int. J. Comput. Appl., vol. 40, no. 15, pp. 19-25, 2012.

[2] D. R. Raymond and S. F. Midkiff, "Denial-of-service in wireless sensor networks: Attacks and defenses," IEEE Pervasive Comput., vol. 7, no. 1, pp. 74-81, 2008.

[3] D. R. Raymond and S. F. Midkiff, "Clustered Adaptive Rate Limiting: Defeating Denial-Of-Sleep Attacks In Wireless Sensor Networks," IEEE, pp. 1-7, 2007.

[4] D. E. Boubiche and A. Bilami, "A Defense Strategy against Energy Exhausting Attacks in Wireless Sensor Networks," J. Emerg. Technol. web Intell., vol. 5, no. 1, pp. 18-27, 2013.

[5] M. Brownfield, Y. Gupta, and N. Davis IV, "Wireless sensor network denial of sleep attack," Proc. of 6th Annual IEEE Syst. Man Cybern. Inf. Assur. Work. SMC 2005, vol. 2005, pp. 356-364, June, 2005.

[6] M. Doudou, D. Djenouri, N. Badache, and A. Bouabdallah, "Journal of Network and Computer Applications Synchronous contention-based MAC protocols for delaysensitive wireless sensor networks: A review and taxonomy," J. Netw. Comput. Appl., vol. 38, pp. 172-184, 2014.

[7] J. Rezaei, "Best-worst multi-criteria decision-making method," Omega, vol. 53, pp. 49-57, 2015.

[8] L. Xu and J. Yang, "Introduction to multi-criteria decision making and the evidential reasoning approach," Isbn, no. 106, pp. 1-21, 2001.

[9] M. I. Brownfield, M. I. Brownfield, and N. J. Davis, "Energy-efficient Wireless Sensor Network MAC Protocol Energy-efficient Wireless Sensor Network MAC Protocol," Management, 2006.

[10] M. Pirretti, S. Zhu, N. Vijaykrishnan, P. Mcdaniel, M. Kandemir, and R. Brooks, "The Sleep Deprivation Attack in Sensor Networks: Analysis and Methods of Defense," Int. J. Distrib. Sens. Networks Int. J. Distrib. Sens. Networks Int. J. Distrib. Sens. Networks, vol. 2, no. 3, pp. 267-287, 2006.

[11] C. Chen, L. Hui, Q. Pei, L. Ning, and P. Qingquan, "An effective scheme for defending denial-of-sleep attack in wireless sensor networks," in 5th International Conference on Information Assurance and Security, IAS 2009, 2009.

[12] T. Bhattasali and R. Chaki, "AMC Model for Denial of Sleep Attack Detection,” arXiv Prepr. arXiv1203.1777, 2012.

[13] R. Falk and H. J. Hof, "Fighting insomnia: A secure wakeup scheme for wireless sensor networks," Proc. 2009 3rd Int. Conf. Emerg. Secur. Information, Syst. Technol. Secur. 2009, pp. 191-196, 2009.

[14] S. Naik and N. Shekokar, "Conservation of energy in wireless sensor network by preventing denial of sleep attack," in Procedia Computer Science, 2015.

[15] C. T. Hsueh, C. Y. Wen, and Y. C. Ouyang, "A secure scheme against power exhausting attacks in hierarchical wireless sensor networks," IEEE Sens. J., vol. 15, no. 6, pp. 3590-3602, 2015.

[16] W. Dargie and C. Poellabauer, Fundamentals of Wireless Sensor Networks: Theory and Practice. 2010.

[17] B. A. Networks, “User's Manual,” March, 2011.

[18] S. Isaiadis and V. Getov, "Integrating Mobile Devices into the Grid : Design Considerations and Evaluation," Proc. of Euro-Par 2005 Conference, LNCS, vol. 3648, pp. 10801088, Springer, 2005.

[19] A Merlo, M Migliardi, and L Caviglione, "A survey on energy-aware security mechanisms", Pervasive and Mobile Computing, vol. 24, pp. 77-90, 2015. 